\title{
Nutritional and Micronutrient Status of Elderly People Living in a Rural Community of Bangladesh
}

Sumon Kumar Das ${ }^{1}$, Abu Syed Golam Faruque ${ }^{1 *}$, Shahnawaz Ahmed ${ }^{1}$, Abdullah Al Mamun², Rubhana Raqib ${ }^{1}$, Anjan Kumar Roy ${ }^{1}$, Mohammod Jobayer Chisti ${ }^{1}$, Tahmeed Ahmed ${ }^{1}$ and Mohammed Abdus Salam ${ }^{1}$

${ }^{1}$ International Centre for Diarrhoeal Disease Research, Bangladesh

${ }^{2}$ School of Population Health, University of Queensland, Brisbane, Australia

\begin{abstract}
Background: To assess the nutrition and micronutrient status of elderly population in the rural area of Bangladesh.

Method: Healthy 44 elderly ( $\geq 60$ years) and 88 middle aged (40-59 years) men and women were studied during April-September 2010. Their anthropometric status, micronutrient status and biochemical markers were assessed.

Result: Mean body mass index (BMI), hemoglobin, alanine transaminase (ALT), albumin, vitamin B12, and fasting blood sugar (FBS) were significantly lower and serum creatinine, vitamin D and folate were significantly higher among elderly compared to that of middle aged population. However, uric acid (UA), calcium, zinc, and retinol were identical in both the groups. Individual being elderly, impacted on decreased level of hemoglobin ( $0.67 \mathrm{gm} / \mathrm{dl})$, vitamin B12 (38.76 pmol/L), albumin $(0.12 \mathrm{gm} / \mathrm{dL})$, zinc $(0.03 \mathrm{mg} / \mathrm{L})$ and FBS $(0.88 \mu \mathrm{mol} / \mathrm{L})$ after adjusting for covariates.
\end{abstract}

Conclusion: Elderly had compromised nutritional status with lower levels of hemoglobin, FBS, vitamin and micronutrient.

Keywords: Elderly; Micronutrient; Nutrition; Rural; Vitamin

\section{Introduction}

Life expectancy is increasing globally, resulting in increased number of ageing population. There were an estimated 400 million people aged 60 years or older in developing countries in 2002, which will increase to approximately 840 million by 2025 (i.e. more than double in just over two decades) representing $70 \%$ of all elderly individuals worldwide [1]. Of all elderly people worldwide, over half of them live in Asia [1]. There is an increased prevalence of micronutrient deficiency in addition to chronic diseases among elderly individuals [2-5]. Moreover, their associated health care costs pose one of the major challenges of the 21st century.

It had been reported that at least $1 \%$ of the healthy elderly individuals at community level suffer from malnutrition which increased from $20 \%$ to $37 \%$ among those who required hospitalization or institutionalized care [6]. Low food intake or consumption of compromised dietary variety by the elderly with poor appetite and difficulty in mastication may lead elderly more vulnerable to malnutrition and micronutrient deficiency. Such health disorders are further accentuated by social, psychological and physical factors [7-9]. Several studies have been conducted to address the overall nutritional status of elderly both in developed [10] as well developing countries [11-13]. However, in most of the developing countries including Bangladesh, maternal and child nutrition $[14,15]$ are still the main where elderly nutrition remains at the bottom of the priority list. The average life expectancy at birth in Bangladesh has increased to over 60 year resulting increase in number of elderly [16]. Although, a small proportion (around 6\%) of the total population of Bangladesh constitutes as elderly, but the absolute number of them is quite significant (about 7.2 million) and the rate of their increase is fairly high [17]. A recent study aimed to assess the health profiles of elderly people of Bangladesh revealed that about $56 \%$ of the elderly population was in average state of health and only 20 percent were not in good health [18]. Another study demonstrated that only $40 \%$ of the elderly individuals had a Body Mass Index (BMI) within the optimal range (18.5-24.9 kg/m2) and at least half of elderly women were chronic energy deficient [19]. There is also lack of evidence-based information on micronutrient deficiencies in elderly people. Thus, our study aimed to assess the overall nutrition and micronutrient status of elderly people living in a rural community in Bangladesh and compare those results with that of middle-aged ( $40-59$ years) people.

\section{Materials and Methods}

\section{Study site}

The study was conducted in a rural community of Mirzapur sub-district under Tangail district of rural Bangladesh from April to September 2010. The study site was located about 40 miles northwest of Dhaka, the capital city of Bangladesh. International Centre for Diarrhoeal Disease Research, Bangladesh (ICCDR, B) has established a Demographic Surveillance System (DSS) in this field site (ASG Faeuque, personal communication). About $30 \%$ of the DSS populations are $\geq 40$ years and $10 \%$ are $\geq 60$ years of age. A tertiary hospital with 750 beds popularly known as Kumudini Hospital is located in the centre of the field site and the facility serves the DSS residents along with individuals reporting from other sub-districts of rural Bangladesh.

\section{Study design, selection and sample size of study participants}

The study was a cross-sectional one; however, we followed a casecontrol design for analysis of data. We defined 'elderly' as those aged 60 years or older based on WHO criteria, and they were our cases. Individuals aged 40-59 years were defined as 'middle aged' (Collins Concise Dictionary) that were considered to represent the comparison group. All study participants were randomly selected from apparently

${ }^{*}$ Corresponding author: Abu Syed Golam Faruque, Center for Nutrition and Food Security, International Centre for Diarrhoeal Disease Research, Bangladesh,Tel: 880-2-8860523-32; Fax: 880-2-9860704; E-mail: gfaruque@icddrb.org

Received May 24, 2012; Accepted June 21, 2012; Published June 25, 2012

Citation: Das SK, Golam Faruque AS, Ahmed S, Mamun AA, Raqib R, et al. (2012) Nutritional and Micronutrient Status of Elderly People Living in a Rural Community of Bangladesh. J Gerontol Geriat Res 1:107. doi:10.4172/2167-7182.1000107

Copyright: ( 2012 Das SK, et al. This is an open-access article distributed under the terms of the Creative Commons Attribution License, which permits unrestricted use, distribution, and reproduction in any medium, provided the original author and source are credited. 
healthy residents of the DSS area. After excluding evidences of respiratory and renal function problems, potential participants were asked for their current or previous history of chronic illnesses such as hypertension (assessed by measuring blood pressure), diabetes mellitus (detected based on fasting blood sugar level) and ischemic heart disease (assessed by signs and symptoms consistent with ischemic heart disease). Participants were enrolled if they provided written informed consent.

Review of global published data indicated a prevalence of micronutrient deficiency among elderly to be $20 \%$ [20] with a margin of error of $12 \%$ and 1.96 for $95 \%$ confidence interval; therefore, the estimated sample size was 43 per group, which had enough power to detect any statistical difference in the outcome. Due to difficulties in finding adequate numbers of healthy elderly, we decided to enroll elderly (60 years and above) and the middle aged ( $40-59$ years) in a ratio of 1:2 to increase the power of the study. From a list of randomly selected 300 individuals aged 40 years and above, we finally enrolled first 44 healthy elderly (case) and first 88 healthy middle aged (control) participants who met the inclusion criteria.

\section{Blood sampling and laboratory measure}

The height and weight of all the participants were measured with locally made wooden height scale and digital weighing scale (TANITAHG314) respectively in the Kumudini Hospital and Body Mass Index (BMI) was calculated $(\mathrm{kg} / \mathrm{m} 2)$. Five milliliters $(5.0 \mathrm{ml})$ of venous blood was collected before 9:00 am following an overnight fasting after a light fat free evening meal. For hemoglobin $0.5 \mathrm{ml}$ blood was put into an Eppendorf tube containing Ethylenediaminetetraacetic acid (EDTA) and, serum was separated. All specimens were transported immediately to the Nutritional Biochemistry Laboratory of icddr,b in Dhaka for the biochemical analyses of: hemoglobin, serum uric acid (Roche), alanine transaminase (ALT), creatinine, calcium, albumin, retinol, vitamin B12 (IMMULITE), folic acid (IMMULITE), 25-hidroxy vitamin D (vitamin D) (IDS) and zinc (AAS) following standard laboratory procedures. Fasting blood sugar (FBS) was determined by a bedside glucometer (ACCU-CHEK, Active, Roche) at the time of blood collection.

\section{Statistical analysis}

Data analysis was done using Statistical Package for Social Sciences (SPSS) Windows (Version 15.2; Chicago, IL) and Epi Info (Version 6.0, USD, Stone Mountain, GA). We performed Student's t test for all continuous variables and in case of non-normal distribution of data, equivalent non-parametric test was applied. Probability of $<0.05$ was considered to be statistically significant and their $95 \%$ confidence intervals (CI) were also equated. Finally, regression method was performed for vitamins and minerals as dependent variables separately to find any association with age as independent variable and other biochemical markers and socio-demographic factors as confounders that might influence the outcome.

\section{Results}

Both the study groups were enrolled considering the identical socio-demographic characteristics. However, they vary with their occupation; $57 \%$ of male elderly were farmer as opposed to $30 \%$ for middle aged, where as $10 \%$ elderly and $30 \%$ middle aged were involved with small business. On the other hand most of the female participants were housewife. Twenty five percent individuals of both the groups had history of smoking (data not shown). BMI and blood hemoglobin, FBS, serum albumin, ALT and vitamin B12 were significantly lower while serum creatinine and folic acid were significantly higher among elderly individuals compared to middle aged participants (Table 1). However parameters such as serum uric acid, retinol, vitamin D and zinc were similar in both the age groups.

In univariate analysis; haemoglobin, vitamin B12, albumin, zinc and FBS were found to be negatively impacted being individual elderly; however folic acid was positively associated with aging. In multivariate analysis, considering the vitamins and micronutrients separately as dependent variable, similar finding was observed after adjusting for BMI, ALT, creatinin, uric acid, wealth index, physical exercise and sex. Individual being elderly impacted on decreased level of hemoglobin (0.67 gm/dl), Vitamin B12 (38.76 pmol/L), albumin (0.12 gm/dL), zinc $(0.03 \mathrm{mg} / \mathrm{L})$ and FBS $(0.88 \mu \mathrm{mol} / \mathrm{L})$ in rural population (Table 2$)$. However, age was not associated with retinol, folic acid, calcium and vitamin $\mathrm{D}$ neither in univariate nor in multivariate models (Table 2).

\section{Discussion}

Results of our study indicated that the elderly individuals of Bangladesh had lower levels of different vitamins and micronutrients as well as compromised regulatory and metabolic functions compared to their middle aged counterparts. Elderly had lower hemoglobin, ALT, albumin, vitamin B12, and fasting blood sugar; and higher serum creatinine and folate level. We found significant negative association with hemoglobin, albumin, vitamin B12 and FBS in elderly.

In the present study, elderly had lower mean hemoglobin level compared to middle aged. Compromised digestive function among elderly may have contributed to such lower levels [21]. Several studies have reported a notable increase in prevalence of anemia in the oldest individuals and men had higher the threshold value than women [22]; however, we did not observe any significant difference in anemia between the groups, this could be due to lack of sufficient study power for this analysis. In this study, older aged individuals had significant lower hemoglobin level and such observations have been reported by previous studies [23]. We also found similar correlation with vitamin B12 like earlier studies [24]. It could be a mixed effect, as vitamin $\mathrm{B} 12$ is one of the important components in haemopoiesis. Any kind of its deficiencies is likely to result in anemia. However, it has been estimated that $10-15 \%$ of people aged 60 years and more suffer from vitamin B12 deficiency much more in developing countries due to low consumption of animal-source foods $[20,25]$. Gastric atrophy in

\begin{tabular}{|c|c|c|c|}
\hline Indicators & Elderly, $(n=44)$ & Middle aged, $(n=88)$ & $\mathrm{p}$-value \\
\hline BMI & $19.26 \pm 2.80$ & $21.07 \pm 3.21$ & 0.01 \\
\hline Hemoglobin (gm/dL) & $12.28 \pm 1.65$ & $12.90 \pm 1.65$ & 0.04 \\
\hline S. Creatinine $(\mu \mathrm{mol} / \mathrm{L})$ & $67.98 \pm 17.77$ & $58.83 \pm 1.78$ & 0.03 \\
\hline S. ALT (U/L) & $13.41 \pm 8.26$ & $20.40 \pm 10.51$ & $<0.01$ \\
\hline S. Uric acid $(\mu \mathrm{mol} / \mathrm{L})$ & $270 \pm 81.42$ & $271.66 \pm 70.63$ & 0.94 \\
\hline S. Retinol ( $\mu \mathrm{g} / \mathrm{L})$ & $40.67 \pm 11.44$ & $37.61 \pm 10.00$ & 0.12 \\
\hline S. Vitamin B12 (pmol/L) & $150.25 \pm 61.26$ & $194.14 \pm 105.15$ & $<0.00$ \\
\hline S. Folic acid (nmol/L) & $22.43 \pm 9.51$ & $18.66 \pm 11.71$ & 0.01 \\
\hline S. Calcium (mg/dL) & $9.35 \pm 0.50$ & $9.22 \pm 0.44$ & 0.14 \\
\hline S. Albumin (gm/dL) & $4.18 \pm 0.28$ & $4.33 \pm 0.28$ & $<0.01$ \\
\hline $\begin{array}{l}\text { S. 25-hidroxy vitamin D } \\
\text { (nmol/L) }\end{array}$ & $70.98 \pm 16.85$ & $60.91 \pm 22.88$ & 0.01 \\
\hline S. Zinc (mg/L) & $0.86 \pm 0.16$ & $0.92 \pm 0.20$ & 0.06 \\
\hline FBS $(\mu \mathrm{mol} / \mathrm{L})$ & $5.19 \pm 1.21$ & $6.18 \pm 1.57$ & $<0.01$ \\
\hline
\end{tabular}

Abbreviations: BMI, body mass index; ALT, alinine transaminase; FBS, fasting blood sugar Values are mean $\pm S d$, unless specified otherwise; $S$, serum * P-value indicates the significance level of the mean difference of indicators between elderly and middle aged group. We used two independent group mean comparison test.

Table 1: Nutrition, vitamins and micronutrients status of elderly and middle aged. 


\begin{tabular}{|l|c|c|}
\hline & $\begin{array}{c}\text { Unadjusted mean } \\
\text { difference }(95 \% \mathrm{Cl})\end{array}$ & $\begin{array}{c}\text { Adjusted mean difference } \\
(95 \% \mathrm{Cl})\end{array}$ \\
\hline Hemoglobin $(\mathrm{gm} / \mathrm{dl})$ & $-0.62(-1.23,-0.01)$ & $-0.67(-1.29,-0.05)$ \\
\hline S. Retinol $(\mu \mathrm{gg} / \mathrm{L})$ & $3.05(-0.78,6.89)$ & $4.10(-0.24,8.45)$ \\
\hline S. Vitamin B12 $(\mathrm{pmol} / \mathrm{L})$ & $-43.88(-77.87,-9.90)$ & $-38.76(-74.45,-3.06)$ \\
\hline S. Folic acid $(\mathrm{n} \mathrm{mol} / \mathrm{L})$ & $3.77(-0.34,7.89)$ & $3.93(-0.58,8.45)$ \\
\hline S. Calcium $(\mathrm{mg} / \mathrm{L})$ & $0.12(-0.04,0.29)$ & $0.07(-0.12,0.26)$ \\
\hline S. Albumin $(\mathrm{gm} / \mathrm{dL})$ & $-0.15(-0.25,-0.05)$ & $-0.12(-0.24,-0.01)$ \\
\hline S. $25-$ hidroxy vitamin D \\
(nmol/L) & $10.07(2.22,17.92)$ & $8.23(-0.32,16.77)$ \\
\hline S. Zinc $(\mathrm{mg} / \mathrm{L})$ & $-0.06(-0.13,-0.00)$ & $-0.03(-0.12,-0.05)$ \\
\hline FBS $(\mu \mathrm{mol} / \mathrm{L})$ & $-0.99(-1.52,-0.45)$ & $-0.88(-1.48,-0.29)$ \\
\hline
\end{tabular}

- Outcome variables: Hemoglobin, retinol, vitamin B12, folic acid, calcium, albumin, vitamin D, zinc, Fasting blood sugar (FBS).

- Main exposure: age group, middle age vs. elderly (middle age is the reference category)

- Adjusted factors: body mass index (BMI), alinine transaminase (ALT), S. creatinin, uric acid, wealth index, physical exercise and sex.

Table 2: Un adjusted and adjusted mean differences (95\% confidence interval) of different vitamins and minerals between middle aged and elderly.

elderly leads to achlorhydria, low serum pepsinogen I concentration and high serum gastrin level which might play important role in such a deficiency $[20,21]$. Additionally, dyspepsia, often reported by elderly may contribute to the deficiency state. Again folate, another important micronutrient is as important as Vitamin B12 and its deficiency is also common in elderly people [26]. However, we neither found any lower level nor any association with older age.

The current study showed significant association between older age and lower serum albumin which resembled that of the previous findings [27]. Age-related changes such as metabolic disorders, inadequate dietary intake, physical activity or disability and altered body composition are thought to be influenced by the serum albumin [27]. Other than that elderly are more vulnerable to trauma and infection which may influence in rapid reduction of serum albumin [27]. Studies have described an association between low serum albumin concentration and increasing morbidity and mortality [28].

Interestingly we noted lower levels of FBS among elderly compared to the middle aged and an association was revealed in regression analysis. Individuals with hyperglycemia were not included into the study at the time of enrollment, and we failed to identify any factors that might explain this finding. However, advanced aging is likely to be manifested with decreased appetite [29], reduced optimum digestive mechanisms of the gastrointestinal tract and improper absorption of the ingested food products after digestion by the intestinal brush border enzymes which may explain lower level of blood glucose levels among elderly individuals.

We considered all healthy individuals as cases as well as their corresponding comparison group members with normal hepatic function and without any renal impairment. It has been documented that ageing along with altered hepatic functions including gluconeogenesis, and reduced serum ALT might reflect such changes in the liver [30]. We also observed low ALT levels among rural elderly; however, it was within the normal range. Our elderly individuals had higher mean serum creatinine $(67.98 \mu \mathrm{mol} / \mathrm{L})$ which was also within normal limit. Though we excluded renal impairment, higher mean levels might be an early manifestation of renal function disorders as aging results in sclerosis of glomeruli of kidney which may be reflected by higher concentrations of serum creatinine [31]. We also considered normal serum uric acid that plays an important role as predictor for various chronic diseases like cardiovascular diseases [32], hypertension
[33], chronic kidney diseases [34], and diabetes mellitus [35] among study individuals. Moreover, we excluded overweight and underweight individuals as both were associated with different micronutrient deficiencies [36], even though elderly had lower mean BMI which could be due to reduced body muscle mass [19].

The present study failed to detect any association between zinc and increasing age. Previous studies have reported zinc deficiency as more common in elderly than younger people irrespective of sex [37]. This might be related to less intake of high zinc containing food especially meat and intake of higher phytate containing meal e.g. rice based diet by the elderly $[37,38]$. So as for vitamin $\mathrm{D}$, elderly also have lower serum vitamin D than the middle aged [39]. Due to limited exposure to sunlight, consequent to lesser outdoor activities might be a contributor to vitamin D deficiency among the elderly. On the other hand, vitamin $\mathrm{D}$ is one of the major calcium regulatory components of the body [40]. Thus, its imbalance generally reflects in calcium deficiency as well [41], nonetheless, our observations correspond to previous findings. We noted serum retinol to be similar in both groups without any significant association, although elderly have been reported to be more likely to have retinol deficiency [42].

In the present study, rural elderly had lower content of vitamin and minerals. Several factors might explain our observations. Aging may be a consequence of oxidation that damages DNA, protein, carbohydrates and lipids and leads to degenerative diseases due to a disruption of cellular homeostasis [43]. Simultaneously, inadequate intake attributable to a lack of appetite or difficulty in preparing food, depression, isolation, low income and reduced sense of smell, taste, drug-nutrient interaction and reduction in nutrient absorption contribute to malnourishment among the elderly [44]. Other than that, achlordydria or hypochlorhydria, is commonly associated with aging $[45,46]$. Age may be associated with a change in intestinal microflora, such as a decrease in anaerobes and bifidobacteria and an increase in enterobacter, reducing intestinal immunity favoring gastrointestinal infection [47]. Recent study reported that $46 \%$ of the physically sound elderly are jobless and $15 \%$ are not engaged in job due to lack of physical fitness [18]. Advanced age belonging to individuals of poor households reported poor health which was significantly associated with lower health-related quality of life (HRQoL) scores in both Bangladesh and many developing countries [48].

It was difficult to enroll healthy elderly individuals because they often suffer from various health disorders. So to conduct the study with reduced number of elderly while having sufficient power of the study, we had to include more than one individual for each elderly study participant in the comparison group. Thus, smaller sample size, might have resulted in any statistically non-significant association. On the other hand, the present study failed to observe any association between few vitamins (retinol, folic acid and vitamin D) and mineral (calcium), and age in univariate as well as multivariate analysis, which was observed in the other studies $[26,39,42]$. We do not have any ready explanation for such findings; this might be associated with dietary habits or exposure to sunlight. The present study did not collect information on dietary habits or perceptions regarding food consumption and health status in that population which might have influenced their nutritional and micronutrient status. On the other hand, the present cross-sectional design failed to capture the changes of nutrition, vitamins and micronutrient status among rural elderly; thus, findings from a rural area might not generalize the rural population of Bangladesh. 


\section{Conclusion}

Rural elderly are more likely to suffer from compromised nutrition status and micronutrient malnutrition. With increasing age, regulatory functions of the vital organs of the body decreases; multiple sociodemographic factors may also influence these functions. It is essential to identify the predictors for such a lower micronutrient status and also need to know the micronutrient level among urban population to address the knowledge gap. Moreover, being conducted only in one sub-district in rural Bangladesh, the study may not be representative of elderly population of Bangladesh and thus further studies are needed in larger population from diverse geographical areas to reflect a representative sample with individuals from different socio-cultural background.

\section{Acknowledgment}

This research study was funded by Government of the People's Republic of Bangladesh through IHP-HNPRP, grant number GR/00410. icddr,b acknowledges with gratitude the commitment of Government of the People's Republic of Bangladesh to its research efforts. We also sincerely acknowledge the support of the Kumudini Women's Medical College and Hospital and, sincerely thank Dr. Dinesh Mondal, Dr. Jena Derakhshani Hamadani, Dr. Sabrina Rasheed and Dr. Fatema Khatun for their valuable comments and suggestions, and we are also thankful to Bidduth Sarkar for screening and specimen collection.

\section{References}

1. WHO (2002) Report of the World Health Organization. Active ageing: a policy framework. Aging Male 5: 1-37.

2. Afridi HI, Kazi TG, Kazi N, Shah AQ, Khan S, et al. (2012) Evaluation of calcium, magnesium, potassium, and sodium in biological samples (scalp hair, serum, blood, and urine) of Pakistani referents and arthritis patients of different age groups. Clin Lab 58: 7-18.

3. Ringbaek T, Martinez G, Durakovic A, Thogersen J, Midjord AK, et al. (2011) Vitamin d status in patients with chronic obstructive pulmonary disease who participate in pulmonary rehabilitation. J Cardiopulm Rehabil Prev 31: 261-267.

4. Roozbeh J, Sharifian M, Ghanizadeh A, Sahraian A, Sagheb MM, et al. (2011) Association of zinc deficiency and depression in the patients with end-stage renal disease on hemodialysis. J Ren Nutr 21: 184-187.

5. Russell RM, Suter PM (1993) Vitamin requirements of elderly people: an update. Am J Clin Nutr 58: 4-14

6. Guigoz Y, Lauque S, Vellas BJ (2002) Identifying the elderly at risk for malnutrition. The Mini Nutritional Assessment. Clin Geriatr Med 18: 737-757.

7. Donini LM, Savina C, Cannella C (2003) Eating habits and appetite control in the elderly: the anorexia of aging. Int Psychogeriatr 15: 73-87.

8. Wham CA, Teh RO, Robinson M, Kerse NM (2011) What is associated with nutrition risk in very old age? J Nutr Health Aging 15: 247-251.

9. Feldblum I, German L, Castel H, Harman-Boehm I, Bilenko N (2007) Characteristics of undernourished older medical patients and the identification of predictors for undernutrition status. Nutr J 6: 37

10. Woo J (2011) Nutritional strategies for successful aging. Med Clin North Am 95: 477-493.

11. Chilima DM, Ismail SJ (1998) Anthropometric characteristics of older people in rural Malawi. Eur J Clin Nutr 52: 643-649.

12. Pieterse S, Manandhar M, Ismail S (1998) The nutritional status of older Rwandan refugees. Public Health Nutr 1: 259-264.

13. Manandhar MC (1999) Undernutrition and impaired functional ability amongst elderly slum dwellers in Mumbai, India. University of London.

14. Kabir I, Khanam M, Agho KE, Mihrshahi S, Dibley MJ, et al. (2012) Determinants of inappropriate complementary feeding practices in infant and young children in Bangladesh: secondary data analysis of Demographic Health Survey 2007. Matern Child Nutr 1: 11-27.

15. Streatfield PK, Koehlmoos TP, Alam N, Mridha MK (2008) Mainstreaming nutrition in maternal, newborn and child health: barriers to seeking services from existing maternal, newborn, child health programmes. Matern Child Nutr 1: $237-255$.

\section{6. http://www.who.org}

17. http://www.banglapedia.com

18. Uddin MT, Chowdhury MAI, Islam MN, Baher* GU (2010) Status of elderly people of Bangladesh: Health Perspective Proc. Pakistan Acad. Sci. 47: 181189.

19. Faruque AS, Khan Al, Roy CN, Malek MA, Salam MA, et al. (2006) Anthropometric characteristics of elderly people: observations at a large diarrheal hospital in Dhaka, Bangladesh. Southeast Asian J Trop Med Public Health 37: 784-792.

20. Baik HW, Russell RM (1999) Vitamin B12 deficiency in the elderly. Annu Rev Nutr 19: 357-377.

21. Allen LH (2008) Causes of vitamin B12 and folate deficiency. Food Nutr Bul 29: S20-34.

22. Beghe C, Wilson A, Ershler WB (2004) Prevalence and outcomes of anemia in geriatrics: a systematic review of the literature. Am J Med 116.

23. Gabrilove J (2005) Anemia and the elderly: clinical considerations. Best Pract Res Clin Haematol 18: 417-422.

24. Loikas S, Koskinen P, Irjala K, Lopponen M, Isoaho R, et al. (2007) Vitamin B12 deficiency in the aged: a population-based study. Age Ageing 36: 177-183.

25. Allen LH (2009) How common is vitamin B-12 deficiency? Am J Clin Nutr 89 693S-696S.

26. Wald NJ, Law M, Hoffbrand VA (2004) Vitamin B-12 and folate deficiency in elderly persons. Am J Clin Nutr 79: 338

27. Gom I, Fukushima H, Shiraki M, Miwa Y, Ando T, et al. (2007) Relationship between serum albumin level and aging in community-dwelling self-supported elderly population. J Nutr Sci Vitaminol(Tokyo) 53: 37-42.

28. Fried LP, Kronmal RA, Newman AB, Bild DE, Mittelmark MB, et al. (1998) Risk factors for 5-year mortality in older adults: the Cardiovascular Health Study. JAMA 279: 585-592.

29. Di Francesco V, Fantin F, Omizzolo F, Residori L, Bissoli L, et al. (2007) The anorexia of aging. Dig Dis 25: 129-137.

30. Ozer J, Ratner M, Shaw M, Bailey W, Schomaker S (2008) The current state of serum biomarkers of hepatotoxicity. Toxicology 245: 194-205.

31. Kaplan C, Pasternack B, Shah H, Gallo G (1975) Age-related incidence of sclerotic glomeruli in human kidneys. Am J Pathol 80: 227-234.

32. Chu NF, Wang DJ, Liou SH, Shieh SM (2000) Relationship between hyperuricemia and other cardiovascular disease risk factors among adult males in Taiwan. Eur J Epidemiol 16: 13-17.

33. Mellen PB, Bleyer AJ, Erlinger TP, Evans GW, Nieto FJ, et al. (2006) Serum uric acid predicts incident hypertension in a biethnic cohort: the atherosclerosis risk in communities study. Hypertension 48: 1037-1042.

34. Sonoda H, Takase H, Dohi Y, Kimura G (2011) Uric Acid levels predict future development of chronic kidney disease. Am J Nephrol 33: 352-357.

35. Wang T, Bi Y, Xu M, Huang $Y, X u Y$, et al. (2011) Serum uric acid associates with the incidence of type 2 diabetes in a prospective cohort of middle-aged and elderly Chinese. Endocrine 40: 109-116.

36. Kimmons JE, Blanck HM, Tohill BC, Zhang J, Khan LK (2006) Association between body mass index and the prevalence of low micronutrient levels among US adults. MedGenMed 8: 59.

37. Yanagisawa H (2008) Zinc deficiency and clinical practice--validity of zinc preparations. Yakugaku Zasshi 128: 333-339.

38. High KP (1999) Micronutrient supplementation and immune function in the elderly. Clin Infect Dis 28: 717-722.

39. Mosekilde L (2005) Vitamin D and the elderly. Clin Endocrinol 62: 265-281.

40. Taylor JG, Bushinsky DA (2009) Calcium and phosphorus homeostasis. Blood Purif 27: 387-394.

41. Riggs BL, Melton LJ 3rd (1986) Involutional osteoporosis. N Engl J Med 314 1676-1686.

42. Nascimento AL, Diniz Ada S, Arruda IG (2007) [Vitamin A deficiency in elderly attending the Health Family Programme in Camaragibe, PE, Brazil]. Arch 
Citation: Das SK, Golam Faruque AS, Ahmed S, Mamun AA, Raqib R, et al. (2012) Nutritional and Micronutrient Status of Elderly People Living in a Rural Community of Bangladesh. J Gerontol Geriat Res 1:107. doi:10.4172/2167-7182.1000107

Latinoam Nutr 57: 213-218.

43. Finkel T, Holbrook NJ (2000) Oxidants, oxidative stress and the biology of ageing. Nature 408: 239-247.

44. Payette H, Boutier V, Coulombe C, Gray-Donald K (2002) Benefits of nutritional supplementation in free-living, frail, undernourished elderly people: a prospective randomized community trial. J Am Diet Assoc 102: 1088-1095.

45. Hurwitz A, Brady DA, Schaal SE, Samloff IM, Dedon J, et al. (1997) Gastric acidity in older adults. JAMA 278: 659-662.
46. Haruma K, Kamada T, Kawaguchi H, Okamoto S, Yoshihara M, et al. (2000) Effect of age and Helicobacter pylori infection on gastric acid secretion. J Gastroenterol Hepatol 15: 277-283.

47. Hebuterne $X(2003)$ Gut changes attributed to ageing: effects on intestina microflora. Curr Opin Clin Nutr Metab Care 6: 49-54.

48. Nilsson J, Rana AM, Luong DH, Winblad B, Kabir ZN (2011) Health-Related Quality of Life in Old Age: A Comparison Between Rural Areas in Bangladesh and Vietnam. Asia Pac J Public Health. 